\title{
CONSERVATIVE AND DIVERGENCE FREE ALGEBRAIC VECTOR FIELDS
}

\author{
E. CONNELL AND J. DROST
}

\begin{abstract}
Suppose $k$ is a field of characteristic 0 and $k^{|n|}=k\left[x_{1} \ldots \ldots, x_{n}\right]$. If $u_{i}$. $f, \in k^{|n|}$ for $1 \leqslant i \leqslant n, 1 \leqslant j \leqslant m, u=\left(u_{1}, \ldots, u_{n}\right)$, the $f$, are relatively prime, and each $f, u$ is conservative, then $u$ is conservative and $\left(f_{1} \ldots \ldots f_{m}\right)$ is unimodular. (Given any $u$ with $|J(u)|=1$, then each derivation $\partial / \partial u$, has divergence 0 . If $D: k^{|n|} \rightarrow k^{|n|}$ is a $k$-derivation with kernel of dimension $n-1$, then there exists a $g$ so that $g D$ has divergence 0 .
\end{abstract}

1. Conservative fields. Throughout this paper, $k$ will denote a field of characteristic $0, R$ will be a commutative ring, and $R^{[n]}$ will be the polynomial ring $R\left[x_{1}, \ldots, x_{n}\right]$. If $u_{i} \in R^{[n]}$ for $1 \leqslant i \leqslant n$, then $u=\left(u_{1}, \ldots, u_{n}\right)$ is called an $n$-dimensional vector field over $R$, or simply an $n$-vector over $R$. An $n$-vector is called conservative provided there exists an $f \in R^{[n]}$ such that $u=\nabla f=\left(\partial f / \partial x_{1}, \ldots \partial f / \partial x_{n}\right)$. If $R$ contains the rational numbers, then $u$ is conservative iff $\partial u_{i} / \partial x_{j}=\partial u, / \partial x_{\text {, for all }}$ $1 \leqslant i, j \leqslant n$. The statement that $f_{1} \ldots, f_{m} \in k^{[n]}$ are relatively prime means that if $g$ divides each $f_{i}$, then $g$ is a unit. They are unimodular if there exists $g_{i}$ such that $\sum f_{i} g_{i}=1$. Unimodular implies relatively prime and if $n=1$, relatively prime implies unimodular.

The following lemma lists the three facts used in proving Theorem 1.2. The first two are well known and the third is just an observation.

LemMa 1.1. Suppose $f_{1}, \ldots, f_{m} \in k^{[n]}$.

(1) Any two of $f_{1} \ldots, f_{m}$ are algebraically dependent over $k$ iff any two of $\nabla f_{1} \ldots, \nabla f_{m}$ are linearly dependent over $k^{[n]}$.

(2) Any two of $f_{1}, \ldots, f_{m}$ are algebraically dependent over $k$ iff there exists $h \in k^{|\prime|}$ such that each $f_{i} \in k[h]$.

(3) Suppose $u$ and $v$ are nonzero n-vectors over $k, h \in k^{[n]}$ is not constant. $g_{1}, \ldots, g_{m} \in k[h]$ are relatively prime in $k[h], f_{1}, \ldots, f_{m} \in k^{[n]}$ are relatively prime in $k^{[n]}$, and $g_{i}(h) v=f_{i} u$ for $i=1, \ldots, m$. Then there exists $t \in(k-0)$ such that $v=t u$ and $\operatorname{tg}_{1}=f_{1}$.

Proof. Part (1) follows Chapter 10 of [M]. Part (2) follows from Theorem 2.11 of [AHE]. Also it is immediate from their results that if $f \in k^{[n]}$ is nonconstant. then there exists $h \in k^{[n]}$ such that $k[h]$ is the algebraic closure of $k[f]$ in $k^{[n]}$. Now suppose the hypothesis of part (3). Then there exists $q_{1} \in k[h]$ such that $\Sigma g_{i} q_{1}=1$.

Received by the editors May 18, 1982 and, in revised form. July 30, 1982. 1980 Mathematics Subject Classification. Primary 13F20: Secondary 13N05. 
Thus $v=\Sigma q_{i} f_{i} u=t u$ where $t \in k^{[n]}$. Substituting gives $g_{i} t u=f_{i} u$. So $t$ divides each $f_{i}$ and thus $t \in k-0$.

THEOREM 1.2. Suppose $u$ is a nonzero n-vector over $k$, and $f_{1} \ldots, f_{m} \in k^{[n]}$ are relatively prime with each $f_{i} u$ conservative. Then $u$ is conservative and the $f_{1}$ are unimodular. Furthermore, there exists an $h \in k^{[n]}$ such that each $f_{l} \in k[h]$.

Proof. Suppose the hypothesis. Then there exist $p_{i} \in k^{[n]}$ with $\nabla p_{1}=f_{1} u$. Since any two of the $\nabla p_{i}$ are linearly dependent over $k^{|n|}$, it follows from part (1) above that any two of the $p_{1}$ are algebraically dependent. From part (2), there exists $h \in k^{[n]}$ such that each $p_{i} \in k[h]$. By a change of notation. let $p_{i}$ be a polynomial in $h, p_{i}(h) \in k[h] \subset k^{[h]}$. Now $p_{i}^{\prime} \nabla h=f_{i} u$. Let $b(h)$ be the g. c. d. of the $p_{i}^{\prime}(h)$ in $k[h]$. $p_{i}^{\prime}(h)=g_{i}(h) b(h)$, and let $v=b(h) \nabla h$. So $g_{i}(h) v=f_{i} u$ for all $i$, and by part (3) above, there exists $t \in k-0$ with $v=t u$. This gives $t u=b(h) \nabla h$ which is conservative because if $c(h)$ is any antiderivative of $b(h), b(h) \nabla h=\nabla c(h)$. Thus $u$ is conservative.

Now $f_{t}=\operatorname{tg}(h)$ and thus each $f_{i} \in k[h]$. Since the $f_{t}$ are relatively prime in $k^{[n]}$. they are relatively prime in $k[h]$, and thus unimodular.

If $u$ is a conservative vector, when is fu conservative? The answer is given below, and the proof does not depend on Theorem 1.2

THEOREM 1.3. Suppose $u=\nabla g$ is a nonzero conservative n-vector over $k, f \in k^{[n]}$. and $k[h]$ is the algebraic closure of $k[g]$ in $k^{[n]}$. Then the following are equivalent:

(1) $f u$ is conservative.

(2) $\nabla f$ and $u$ are linearlv dependent over $k^{[n]}$.

(3) $f$ and $g$ are algebraically dependent over $k$.

(4) $f \in k[h]$.

ProOf. The last three statements are clearly equivalent, and it will be shown that the first two are. Suppose $u$ is conservative. We show $f u$ is conservative iff

$$
\left(\begin{array}{ccc}
u_{1} & \cdots & u_{n} \\
\frac{\partial f}{\partial x_{1}} & \cdots & \frac{\partial f}{\partial x_{n}}
\end{array}\right)
$$

has rank 1 , i.e. every $2 \times 2$ submatrix has determinant 0 . Now $f u$ is conservative iff $\partial\left(f u_{1}\right) / \partial x_{1}=\partial\left(f u_{1}\right) / \partial x_{i}$ which is the same as $u_{i} \partial f / \partial x_{1}=u_{1} \partial f / \partial x_{1}$ and the result follows.

2. Divergence and derivations. If $u$ is an $n$-vector over $R$, the divergence of $u$ is $\operatorname{div}(u)=\partial u_{1} / \partial x_{1}+\cdots+\partial u_{n} / \partial x_{n}$. The following corollary is immediate from Theorem 1.2 and the observation that $u=\left(u_{1}, u_{2}\right)$ has divergence 0 iff $\left(\left(u_{2},-u_{1}\right)-u_{1}\right)$ is conservative.

COROILARY 2.1. If $u=\left(u_{1}, u_{2}\right)$ is a nonzero 2-vector over $k$, and $f_{1} \ldots, f_{m} \in k^{|2|}$ are relatively prime with each $\operatorname{div}\left(f_{i} u\right)=0$, then $\operatorname{div}(u)=0$ and the $f_{1}$ are unimodular.

Question. Suppose $u$ is an $n$-vector over $k$, and $f_{1} \ldots \ldots f_{m} \in k^{|n|}$ are unimodular with $\operatorname{div}(f, u)=0$. What conditions are needed to imply $\operatorname{div}(u)=0$ ? 
If $A$ is a commutative ring, the statement that a function $D: A \rightarrow A$ is a derivation means that $D\left(a_{1}+a_{2}\right)=D\left(a_{1}\right)+D\left(a_{2}\right)$ and $D\left(a_{1} a_{2}\right)=a_{1} D\left(a_{2}\right)+a_{2} D\left(a_{1}\right)$. If $B$ is a square matrix over $A$, the classical adjoint is the matrix $C$ whose $(i, j)$ term is $(-1)^{i+j}$ times the $(j, i)$ minor of $B$. Then $B C=C B=|B| I$ where $I$ is the identity matrix.

TheORem 2.2. Suppose $A$ is a commutative ring, $D_{1}: A \rightarrow A$ is a derivation for $i=1, \ldots, n, D_{i} D_{1}=D_{i} D_{i}, f_{i} \in A$ for $i=1, \ldots, n$ and $e_{i}=(0, \ldots, \ldots, 0)$. Then:

(1) If $v=\left(v_{1}, \ldots, v_{n}\right) \in A^{n}$ is given $b$.

$$
v=|| \begin{array}{ccc}
e_{1} & \cdots & e_{n} \\
D_{1}\left(f_{2}\right) & \cdots & D_{n}\left(f_{2}\right) \\
\vdots & & \vdots \\
D_{1}\left(f_{n}\right) & \cdots & D_{n}\left(f_{n}\right)
\end{array} \mid
$$

then $D_{1}\left(v_{1}\right)+\cdots+D_{n}\left(v_{n}\right)=0$.

(2) If $\left(c_{i, j}\right)$ is the classical adjoint of

$$
D_{j}\left(f_{1}\right)=\left|\begin{array}{ccc}
D_{1}\left(f_{1}\right) & \cdots & D_{n}\left(f_{1}\right) \\
\vdots & & \vdots \\
D_{1}\left(f_{n}\right) & \cdots & D_{n}\left(f_{n}\right)
\end{array}\right|
$$

then for each $1 \leqslant j \leqslant n, D_{1}\left(c_{1, j}\right)+\cdots+D_{n}\left(c_{n, j}\right)=0$.

Proof. To show (1) is to show $D_{1}\left(M_{1,1}\right)-D_{2}\left(M_{1,2}\right)+\cdots \pm D_{n}\left(M_{1, n}\right)=0$ where $M_{1, j}$ is the $(1, j)$ minor. For $n=2$, the theorem states that $D_{1}\left(D_{2}\left(f_{2}\right)\right)-D_{2}\left(D_{1}\left(f_{2}\right)\right)$ $=0$, which is in the hypothesis. In general, it states that

$$
\sum(-1)^{|\sigma|} D_{\sigma(1)}\left(D_{\sigma(2)}\left(f_{2}\right) \cdots D_{\sigma(n)}\left(f_{n}\right)\right)=0,
$$

where the sum is over all permutations $\sigma$ of $(1,2, \ldots, n)$. This is

$$
\begin{aligned}
\sum(-1)^{|\sigma|}\left[D_{\sigma(1)}\left(D_{\sigma(2)}\left(f_{2}\right)\right) D_{\sigma(3)}\left(f_{3}\right)\right. & \cdots D_{\sigma(n)}\left(f_{n}\right) \\
& \left.+\cdots+D_{\sigma(2)}\left(f_{2}\right) D_{\sigma(3)}\left(f_{3}\right) \cdots D_{\sigma(1)}\left(D_{\sigma(n)}\left(f_{n}\right)\right)\right]
\end{aligned}
$$

and it must be seen that these terms cancel in pairs. For simplicity, consider the first term $D_{\sigma(1)}\left(D_{\sigma(2)}\left(f_{2}\right)\right) D_{\sigma(3)}\left(f_{3}\right) \cdots D_{\sigma(n)}\left(f_{n}\right)$ for a given permutation $\sigma$. This term is also given by the permutation $\tau$ where $\tau(1)=\sigma(2), \tau(2)=\sigma(1)$, and $\tau(i)=\sigma(i)$ for $i>2$. Since $\tau$ and $\sigma$ have different signs, the two terms cancel and in this manner, the sum of all terms is zero.

Part (2) follows from part (1) and the definition of classical adjoint, although with this notation it is most easily seen for column 1 of $\left(c_{i, j}\right)$.

A derivation $D: R^{[n]} \rightarrow R^{[n]}$ is an $R$-derivation provided $D(R)=0$. There is a one-to-one correspondence between $n$-vectors over $R$ and $R$-derivations. It sends $u=\left(u_{1}, \ldots, u_{n}\right)$ to $D=u_{1} \partial / \partial x_{1}+\cdots+u_{n} \partial / \partial x_{n}$ where $D\left(x_{i}\right)=u_{i}$ (see [GR,p. 152]). The divergence of $D$ is defined to be the divergence of the corresponding vector $u=\left(D\left(x_{1}\right), \ldots, D\left(x_{n}\right)\right)$. The Jacobian matrix of an $n$-vector $u$ is $J(u)=$ $J\left(u_{1}, \ldots, u_{n}\right)=\left(\partial u_{i} / \partial x_{j}\right)$. 
THEOREM 2.3. If $f_{2}, \ldots, f_{n} \in R^{[n]}$, the $R$-derivation $E: R^{[n]} \rightarrow R^{[n]}$ defined by $E\left(f_{1}\right)=\left|J\left(f_{1}, f_{2}, \ldots, f_{n}\right)\right|$ has divergence 0 .

Proof. This is a restatement of Theorem 2.2(1) with $A=R^{[n]}$ and $D_{i}=\partial / \partial x_{i}$.

THEOREM 2.4. Suppose $f_{i} \in R^{[n]}$ for $1 \leqslant i \leqslant n$ and $f=\left(f_{1}, \ldots, f_{n}\right)$ is the corresponding $n$-vector over $R$. Then:

(1) Each column of the classical adjoint of $J(f)$ has divergence 0 .

(2) If $|J(f)|=1$, each column of $J(f)^{-1}$ has divergence 0 .

(3) Row $i$ of $J(f)$ has divergence 0 iff $f_{i}$ is harmonic, i.e. $\partial^{2} f_{i} / \partial x_{1}^{2}+\cdots+\partial^{2} f_{i} / \partial x_{n}^{2}$ $=0$.

(4) If $R$ is a domain of characteristic 0 , then column $j$ of $J(f)$ has divergence 0 iff the trace of $J(f)$ does not depend on $x_{j}$. Thus the trace of $J(f)$ is constant iff each column of $J(f)$ has divergence 0 .

Proof. Part (1) is a restatement of Theorem 2.2 (2) with $D_{i}=\partial / \partial x_{i}$. Part (2) follows because in this case, the classical adjoint is the inverse. Parts (3) and (4) are just observations.

LEMMA 2.5. (1) If $D: R^{[n]} \rightarrow R^{[n]}$ is an $R$-derivation and $g \in R^{[n]}$, then $\operatorname{div}(g D)=g$ $\operatorname{div} D+D(g)$.

(2) If $g_{i} \in R^{[n]}$ and $D_{i}: R^{[n]} \rightarrow R^{[n]}$ is an $R$-derivation for $i=1, \ldots, m$, and each $D_{i}$ has divergence 0 , then $\operatorname{div}\left(g_{1} D_{1}+\cdots+g_{m} D_{m}\right)=D_{1}\left(g_{1}\right)+\cdots+D_{m}\left(g_{m}\right)$.

Proof. Part (1) is a direct computation and part (2) follows from (1) and the fact that the divergence of the sum is the sum of the divergences.

The divergence of a derivation $D$ on $R^{[n]}$ is defined with respect to the variables $x_{1}, \ldots, x_{n}$. Suppose $f_{1}, \ldots, f_{n}$ is another set of variables, i.e. suppose the algebra homomorphism $R\left[y_{1}, \ldots, y_{n}\right] \rightarrow R\left[x_{1}, \ldots, x_{n}\right]$ which sends $y_{i}$ to $f_{i}$, is an isomorphism. Then any $R$-derivation may be written as $D=u_{1} \partial / \partial f_{1}+\cdots+u_{n} \partial / \partial f_{n}$ where $u_{i}=u_{i}\left(f_{1}, \ldots, f_{n}\right)$. It will be shown below that if $\left|J\left(f_{1}, \ldots, f_{n}\right)\right|=1$, then $\operatorname{div}(D)=$ $\sum \partial u_{i} / \partial f_{i}$.

The Jacobian Conjecture states that if $f=\left(f_{1}, \ldots, f_{n}\right)$ is an $n$-vector over $k$ with $|J(f)|=1$, and $A=k\left[f_{1}, \ldots, f_{n}\right]$, then $A=k^{[n]}$ (see [BCW and Wr]). It is known that any $k$-derivation on $A$ extends to $k^{[n]}$ (see [Wa]). It is shown below that the extension of $\partial / \partial f_{i}$ to $k^{(n)}$ has divergence 0 .

THEOREM 2.6. Suppose $f_{i} \in R^{[n]}$ for $i=1, \ldots, n, f=\left(f_{1}, \ldots, f_{n}\right)$ is the corresponding n-vector over $R,|J(f)|=1$, and $D_{j}$ is the $R$-derivation on $R^{[n]}$ defined by the j-column of $J(f)^{-1}$. Then:

(1) $D_{j}\left(f_{i}\right)=\delta_{i, j}$ and $\operatorname{div}\left(D_{j}\right)=0 . D_{j}$ is written as $D_{j}=\partial / \partial f_{j}$.

(2) If $g_{i} \in R^{[n]}$ for $i=1, \ldots, n$, then $\operatorname{div}\left(g_{1} D_{1}+\cdots+g_{n} D_{n}\right)=D_{1}\left(g_{1}\right)$ $+\cdots+D_{n}\left(g_{n}\right)$.

Proof. For part (1) $D_{j}\left(f_{i}\right)=\delta_{i, j}$ follows from $J(f) J(f)^{-1}=I \cdot \operatorname{div}\left(D_{j}\right)=0$ follows from Theorem 2.4(2). Part (2) follows from (1) and Lemma 2.5(2).

Question. If $A[z]=k^{[n]}$, must $\partial / \partial z$ have divergence 0 ?

Side Comment. Suppose $k^{[n]}$ is unramified over a subalgebra $A[z]$ and the derivation $\partial / \partial z$ on $A[z]$ extends to a derivation on $k^{[n]}$ which does not have 
divergence 0 . Then by Theorem $2.6, A$ is not a polynomial ring in $n-1$ variables. In fact, there could not exist $a_{1}, \ldots, a_{n-1} \in A$ with $d a_{1}, \ldots, d a_{n-1}$ generating the module of differentials $\Omega_{A / k}$ (see [BCW or $\mathbf{W r}$ ] for definitions).

THEOREM 2.7. Suppose $f_{2}, \ldots, f_{n} \in k^{[n]}$ are algebraically independent over $k$ and $D$ and $E$ are nonzero $k$-derivations on $k^{[n]}$ with each $f_{i} \in \operatorname{ker} D \cap \operatorname{ker} E$. Then $D$ and $E$ are linearly dependent, i.e. there exist $s, t \in\left(k^{[n]}-0\right)$ such that $s D=t E$.

Proof. Let $D=\Sigma u_{i} \partial / \partial x_{i}$ and $E=\Sigma v_{i} \partial / \partial x_{i}$. Let $f_{1} \in k^{[n]}$ be such that $f_{1}$, $f_{2}, \ldots, f_{n}$ is a transcendence basis (see $[\mathbf{M}]$ ). Then the $\nabla f_{i}$ are linearly independent, i.e. $J\left(f_{1}, \ldots, f_{n}\right)$ has determinant nonzero and represents an injective homomorphism. Now

$$
\left(\frac{\partial f_{i}}{\partial x_{j}}\right)\left(\begin{array}{c}
u_{1} \\
\vdots \\
u_{n}
\end{array}\right)=\left(\begin{array}{c}
t \\
0 \\
\vdots \\
0
\end{array}\right)
$$

and

$$
\left(\frac{\partial f_{i}}{\partial x_{j}}\right)\left(\begin{array}{c}
v_{1} \\
\vdots \\
v_{n}
\end{array}\right)=\left(\begin{array}{c}
s \\
0 \\
\vdots \\
0
\end{array}\right)
$$

and thus $s u=t v$, i.e. $s D=t E$.

THEOREM 2.8. Suppose $u_{1}, \ldots, u_{n} \in k^{[n]}$ are relatively prime and $D=\Sigma u_{1} \partial / \partial x_{i}$ is a derivation whose kernel has dimension $n-1$. Then there exists a nonzero $g \in k^{[n]}$ such that $\operatorname{div}(g D)=0$.

Proof. Let $f_{2}, \ldots, f_{n} \in k^{[n]}$ be algebraically independent elements in kernel $D$, and $E$ be the derivation defined by $E\left(f_{1}\right)=\left|J\left(f_{1}, \ldots, f_{n}\right)\right|$. Now $E\left(f_{i}\right)=0$ for $i=2, \ldots, n$ by inspection and $\operatorname{div}(E)=0$ by Theorem 2.3. By Theorem 2.7, there exist nonzero $s, t$ such that $s D=t E$, i.e. $s u=t v$ where $v$ is the vector associated with $E$. Since the $u_{i}$ are relatively prime, there exists $g \in\left(k^{[n]}-0\right)$ with $s=\operatorname{tg}$ and so $g D=E$.

THEOREM 2.9. If $D$ is a $k$-derivation on $k^{[n]}$, then its kernel $K$ is algebraically closed in $k^{[n]}$.

Proof. Suppose $b \notin K$ is algebraic over $K$ and $p[T] \in K[T]$ is a polynomial of minimal degree with $p(b)=0$. Then $0=D(p(b))=p^{\prime}(b) D(b)$ gives a contradiction.

THEOREM 2.10. If $D$ is a $k$-derivation on $k^{[2]}$ with $\operatorname{div}(D)=0$, then $\operatorname{ker}(D) \neq k$. Thus if $D$ is not 0 , its kernel has dimension 1 , and is thus equal to $k[h]$ for some $h \in k^{\{2\}}$.

Proof. $D=u_{1} \partial / \partial x_{1}+u_{2} \partial / \partial x_{2}$ and $\partial u_{1} / \partial x_{1}=-\partial u_{2} / \partial x_{2}$. Thus there exists $f \in k^{[2]}$ with $\partial f / \partial x_{1}=-u_{2}$ and $\partial f / \partial x_{2}=u_{1}$. Thus $D(f)=0$. If $\operatorname{ker}(D)$ has dimension 2 , then $D=0$ by Theorem 2.9 . 
ExAmple. $D=x_{1} \partial / \partial x_{1}+x_{2} \partial / \partial x_{2}$ has trivial kernel, and thus there is no $g \in\left(k^{[2]}-0\right)$ so that $\operatorname{div}(g D)=0$.

THEOREM 2.11. If $u_{1}, u_{2} \in k^{[2]}$ are relatively prime and $D=u_{1} \partial / \partial x_{1}+u_{2} \partial / \partial x_{2}$, then the following are equivalent:

(1) $\operatorname{ker}(D)$ has dimension 1 .

(2) There exists a nonzero $g \in k^{[2]}$ with $\operatorname{div}(g D)=0$.

Proof. (1) $\Rightarrow(2)$ by Theorem 2.8 and $(2) \Rightarrow$ (1) by Theorem 2.10.

Questions. If $D$ is $k$-derivation on $k^{[n]}$, what conditions are needed to ensure there exists a nonzero $g$ with $\operatorname{div}(g D)=0$ ? If $\operatorname{div}(D)=0$, what can be said about the kernel of $D$ ?

\section{REFERENCES}

[AHE] S. S. Abhyankar. W. Heinzer and P. Eakin. On the uniqueness of the coefficient ring in a polınomial ring. J. Algebra 23 (1972) 310-342.

[BCW] H. Bass. E. Connell and D. Wright. The Jacohian Conjecture: Reduction of degree and formal expansion of the inverse. Bull. Amer. Math. Soc. (N.S.) 7 (1982), 287-330.

[GR] R. C. Gunning and H. Rossi. Analytic function of seteral complex variahies. Prentice-Hall. Englewood Cliffs. N. J., 1965.

[M] H. Matsumura. Commutative algehra, Benjamin. New York. 1970)

[S] H. M. Schev. Div, Grad, Curl, and all that. Norton. 1973.

[Wa] S. Wang. A Jacohian criterion for separabilit). J. Algcbra 65 (1980), 453-494.

[Wr] D. Wright. On the Jacohian Conjecture, Illinois J. Math. 25 (1981), 423-440).

Department of Mathematics. University of Miami. Coral. (jables. Florida 33124 